\section{Mycobacterium kansasii infection in a psoriasis patient treated with adalimumab and switch to apremilast: First report and literature review}

\author{
Alexandra Maria Giovanna Brunasso, ${ }^{1}$ \\ Sanja Javor, ${ }^{1}$ Emanuele Pontali, ${ }^{2}$ \\ Simona Sola, ${ }^{3}$ Cesare Massone ${ }^{1}$ \\ ${ }^{1}$ Department of Dermatology, \\ ${ }^{2}$ Department of Infectious Diseases, and \\ ${ }^{3}$ Surgical Pathology, Galliera Hospital, \\ Genoa, Italy
}

\begin{abstract}
Patient under anti-TNF-alpha treatment have an increased risk of mycobacterial infections, particularly tuberculosis. Only four case reports of Mycobacterium kansasii infection under anti-TNF- $\alpha$ treatment (two with etanercept, two with infliximab) have been reported, but none under adalimumab. A 72-year-old man treated with adalimumab for psoriasis vulgaris and arthropathic psoriasis, complained on nocturnal cough, occasional hemoptysis and the new onset of ill-defined, reddish, asymptomatic persistent plaques-nodules covered by serum crusts on his back, on the dorsum of the right hand and right middle finger. Routine laboratory investigations, HIV and TB screening (QuantiFERON-TB-Gold test) were all within normal limits. A skin biopsy was inconclusive and special staining resulted negative for microorganisms. Only PCR identified M. kansasii. The patient stopped adalimumab and started anti-TB treatment with gradual improvement of the skin lesions. At 26 months follow-up visit no signs or symptoms of relapse of $M$. kansasii disease occurred.
\end{abstract}

\section{Introduction}

Mycobacterium kansasii (M. kansasii) is primarily a pulmonary pathogen, a nontuberculous mycobacteria (NTM) causing a tuberculosis-like illness particularly in immunocompromised patients. Disseminated infection is uncommon but of poor prognosis therefore an adequate treatment is essential during first manifestations. Cutaneous manifestations in immunocompetent individuals are rare, but they can occur if conditions of altered immunity develop. ${ }^{1,2}$

Anti-tumor necrosis factor alpha (antiTNF- $\alpha$ ) may lead patients to be more susceptible to mycobacterial infections and particularly to tuberculosis

(TB) reactivation. ${ }^{3}$

Herein, we report a psoriasis patient under adalimumab therapy that developed $M$. kansasii infection with skin manifestations. To our knowledge, this is the first report of this association.

\section{Case Report}

A 72-year-old man with a history of recalcitrant psoriasis vulgaris and arthropathic psoriasis, was treated with infliximab $(5 \mathrm{mg} / \mathrm{kg})$ every 8 weeks since 2006 with sustained clinical improvement until 2015 when infliximab lost efficacy (Psoriasis Area and Severity Index: PASI 11.2). Therefore, methotrexate (15 $\mathrm{mg} /$ weekly $+5 \mathrm{mg}$ folic acid) was added concomitantly for 1 year, obtaining only partial improvement. His past medical history recorded hypertension and benign prostate hyperplasia in treatment with olmesartan, hydrochlorothiazide, dutasteride and tamsulosin for years. In April 2016 the patient was switched to adalimumab $40 \mathrm{mg}$ subcutaneously every two weeks with rapid psoriasis improvement. Since September 2016, the patient complained on nocturnal cough and occasional hemoptysis without fever, but ORL- and pneumologyconsultations did not detect any significant alteration. At dermatological follow-up visit in September 2017, the patient complained the new onset of ill-defined, reddish, asymptomatic persistent plaques-nodules covered by serum crusts on his back, on the dorsum of the right hand and right middle finger (Figure 1). Routine laboratory investigations (blood count, liver and renal function, protein $\mathrm{C}$ reactive, erythrocyte sedimentation rate) were all within normal limits. Human immunodeficiency virus (HIV) and TB screening (QuantiFERONTB-Gold test) had been performed yearly with negative results. No risk factors due to his social status or work were relevant; the only daily activity reported was gardening.

Histopathology of a skin biopsy of the lesion on his back performed to rule out persistent herpes infection, deep fungal infection and skin lymphoma showed a micropustular formations within epidermis and a dense lympho-histiocytic inflammatory infiltrate in the dermis. Periodic acid Schiff, Grocott, Ziehl-Neelsen and Alcian blu stainings resulted negative for microorganisms. Skin swab culture was also negative. Polymerase chain reaction (PCR) for Leishmania and Mycobacteria identified M. kansasii. Thorax-TC scan showed mediastinal lymphadenopathies and right
Correspondence: Alexandra Maria Giovanna Brunasso, Dermatology Unit, Galliera Hospital, Via Volta 6, Genoa, Italy.

Tel.: +39.0105634271

E-mail: alexandra.brunasso@galliera.it

Key words: Psoriasis, Adalimumab, QuantiFeron Tb Gold Test, Mycobacteria, Mycobacterium kansasii.

Contributions: The authors contributed equally.

Conflict of interest: The authors declare no potential conflict of interest.

Funding: None.

Ethical approval: Obtained.

Consent for publication: Consent was obtained.

Availability of data and material: Data and materials are available by the authors.

Please cite this article as: Brunasso AMG Javor S, Pontali E, et al. Mycobacterium kansasii infection in a psoriasis patient treated with adalimumab and switch to apremilast: First report and literature review. Dermatol Rep 2021;13:8797.

Received for publication: 16 July 2021. Accepted for publication: 16 November 2021.

This work is licensed under a Creative Commons Attribution-NonCommercial 4.0 International License (CC BY-NC 4.0).

C Copyright: the Author(s), 2021

Licensee PAGEPress, Italy

Dermatology Reports 2021; 13:8797

doi:10.4081/dr.2021.8797

apical fibrosis in the absence of parenchimal lesions, excluding active lung disease. Sputum was three times negative. Infectious disease-specialist consultation diagnosed $M$. kansasii infection based on clinicomolecular correlation. The patient stopped adalimumab and started treatment with isoniazid $300 \mathrm{mg}$ daily, rifampicin $300 \mathrm{mg}$ twice daily, ethambutol $400 \mathrm{mg}$ t.i.d. for 12 months. Therapy was well tolerated without signs of hepatotoxicity.

During anti-mycobacterial treatment, the psoriasis remained stable; cough, hemoptysis and skin lesions slowly improved requiring one year for complete remission (Figure 2). However, at the end of the treatment for $M$. kansasiii, psoriasis relapsed (PASI 6.2) together with joint involvement. Apremilast $30 \mathrm{mg}$ twice daily was started. At 16 weeks follow-up, psoriasis (PASI 2.1) and psoriatic arthritis improved. At 26 months follow-up visit no signs or 
symptoms of relapse of $M$. kansasii disease occurred, apremilast therapy is still ongoing and well tolerated; psoriasis remains stable (PASI 1.8).

\section{Discussion}

M. kansasii is primarily a pulmonary pathogen, causing a TB-like illness (although fever may be less common) that generally manifests in HIV-positive patients and only few reports are associated with iatrogenic immunosuppression; other predisposing conditions include HIV infection, pneumoconiosis, alcoholism, and malignancy. Disseminated infection is uncommon but of poor prognosis, therefore early detection and adequate treatment is mandatory. ${ }^{1,2}$ Tap water is likely the major reservoir for $M$. kansasii infections. Inoculation of NTM into the host usually occurs due to skin injuries in immunocompromised patients and/or associated with conditions of altered immunity. ${ }^{4,5}$ In the United States, infection with $M$. kansasii is the second most common cause of NTM after $M$. avium intracellular infection. ${ }^{6}$

Anti-TNF- $\alpha$ agents are widely used to treat chronic inflammatory diseases; among well-known side effects, they may inhibit the orderly recruitment of cells necessary for the proper granuloma formation and the containment of intracellular pathogens. ${ }^{7}$ Therefore, anti-TNF- $\alpha$ may lead patients to be more susceptible to mycobacterial infections and, in particular, to TB reactivation. ${ }^{7}$ Screening of active and latent TB is mandatory before treatment. ${ }^{3,7}$ Traditionally, TB infection is considered with a greater risk (5-10 times) than NTM infections in patients receiving anti-TNF- $\alpha .^{8}$ However, another study reported that NTM infections were more frequent than TB; among 105 confirmed cases of NTM, $M$. avium intracellulare was accounted for 52 cases $(50 \%)$ whereas only 3 cases of $M$. kansasii $(3 \%){ }^{8}$

NTAM infection should always be considered in the differential diagnosis in patients under anti-TNF- $\alpha$ presenting TBlike symptoms or with new onset of polymorphous persistent cutaneous lesions (erythematous papules and/or nodules and/or plaques, ulcerations, folliculitis). Diagnosis is based on chest imaging with highresolution CT and microbiologic confirmation with sputum testing and highly accurate nucleic acid probes. ${ }^{1,2}$ The American Thoracic Society (ATS) and Infectious Disease Society of America (IDSA) have jointly established specific criteria for the diagnosis of NTM lung disease. ${ }^{9}$ Our patient resulted negative during QuantiFERON-TB Gold test, not surprisingly considering that positive responses can be found only in up to $52 \%$ of cases of M. kansasii infection due to the presence of both early secreted antigenic target-6 (ESAT-6) and culture filtrate proteins (CFP-10) antigens in M. kansasii. ${ }^{10}$

Ziehl-Neelsen staining is often falsely negative in skin biopsy, depending on the mycobacterial index. ${ }^{1}$ In culture, $M$. marinum, M. kansasii, and M. avium- intracellulare are slow-growing mycobacteria (3-4weeks), while $M$. fortuitum, M. chelonaea, and M. abscessus are rapidly growing mycobacteria. ${ }^{1,2,11}$ Since the concentration of organisms may be low, multiple cultures are recommended. Recent studies evidenced that the broad range panmycobacterial PCR can detect all the mycobacterial species directly from clinical specimens with an excellent specificity. ${ }^{12}$ In our patient, the initial diagnosis was based on molecular detection of mycobacterial DNA in skin lesions, then confirmed by

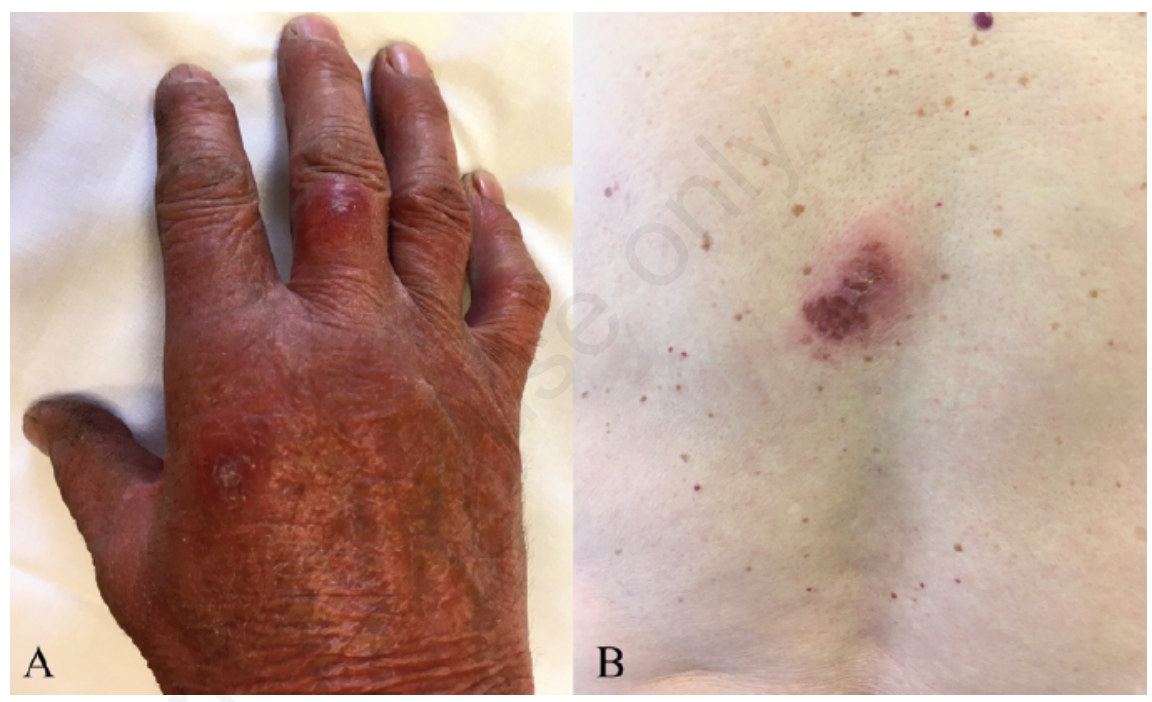

Figure 1. A) Asymptomatic, ill-defined, reddish plaques and nodules on dorsum of the right hand and right middle finger. B) Plaques covered by serum crusts on patient's back.

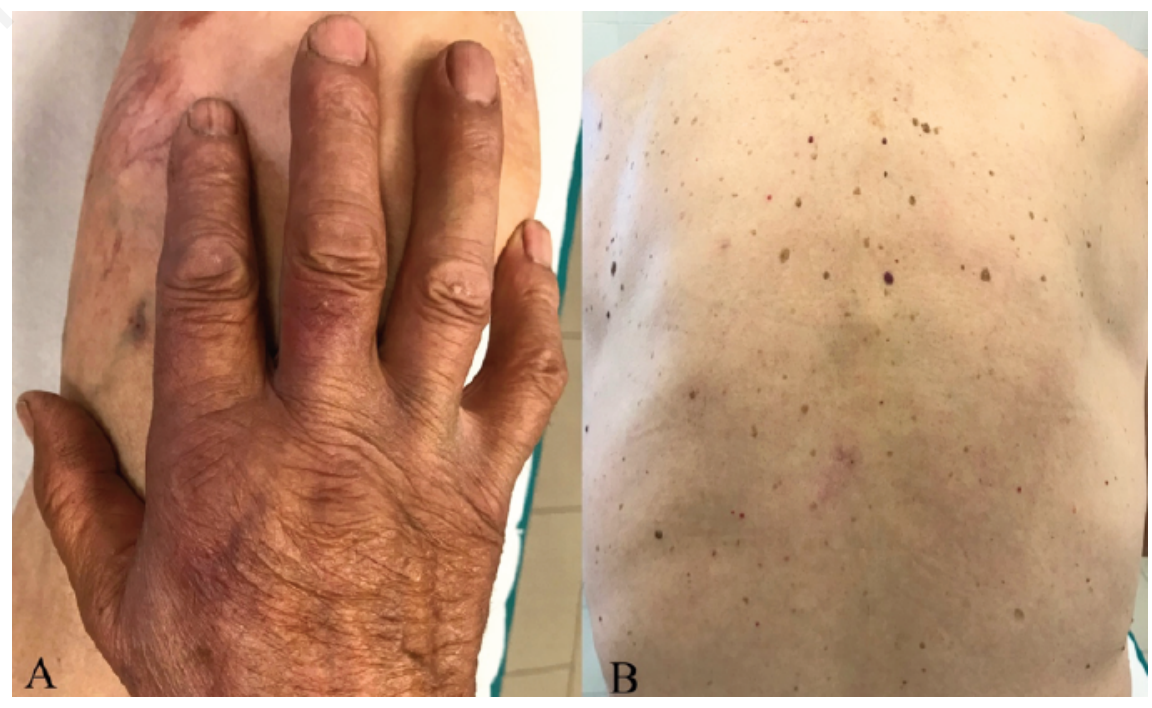

Figure 2. A) Complete resolution of skin lesions with residual dyschromia on dorsum of the right hand and right middle finger $B$ ) Complete resolution of skin lesions on the back at 13-month follow-up. 
resolution of skin lesions and symptoms after prolonged therapy. Mycobacterial DNA identification using PCR is considered not only the most sensitive and specific test to confirm the diagnosis but also the most rapid laboratory test, with a median time to identification of 6 days versus 35 days for culture and which represents a 29-day shorter time to results. ${ }^{11,12}$ Skin manifestation during $M$. kansasii infection are possible and clinical features may vary from nodules, verrucous or ulcerated erythematous plaques, sometimes distributed as sporotrichoid-like lesions, ulcers, cellulitis, abscesses, seromas and even papulo-necrotic tuberculoid-like reactions. ${ }^{13}$ Being immunosuppressed subjects more susceptible, a primary cutaneous infection in immunocompetent individual is rare. ${ }^{4}$

To our knowledge, in the English literature only four case reports of $M$. kansasii infection under anti-TNF- $\alpha$ treatment (two with etanercept, two with infliximab, none with adalimumab) have been reported. ${ }^{6}$ Min and Amlani described a patient with rheumatoid arthritis receiving etanercept that developed M. kansasii lung infection. ${ }^{6}$ Fernández-Guerrero reported a patient with ankylosing spondylitis treated with infliximab and also M. kansasii lung infection isoniazid-resistant. ${ }^{14}$ Spiliopoulou et al. reported a patient with sarcoidosis initially treated with etanercept then switched to infliximab; this patient presented also skin manifestations constituted by confluent vesicles and bullae on the dorsum of the left foot with underlying erythema and oedema. ${ }^{15}$ Malkin et al described an olecranon bursitis secondary to $M$. kansasii infection in a patient receiving infliximab for Behcet's disease. ${ }^{16}$ Additionally, Hsu and coll. reported a 38-year-old woman treated with prednisolone (20 $\mathrm{mg}$ daily), hydroxychloroquine (200 $\mathrm{mg}$ twice daily) and azathioprine (50 $\mathrm{mg}$ twice daily) for a systemic lupus erythematosus who developed M. kansasii cellulitis. ${ }^{17}$

We switched therapy to apremilast due to the efficacy in both skin and joint compartments and the excellent safety profile regarding opportunistic infections. ${ }^{18}$

\section{Conclusions}

In patients receiving anti-TNF- $\alpha$ treatment, physicians should be aware of new onset of persistent and polymorphous skin lesions, and NTM should be always a differential diagnosis. Because histopathology and skin cultures sometimes might not be conclusive, PCR should be performed in all suspicious cases.

\section{References}

1. Griffith DE. Overview of nontuberculous mycobacterial infections in HIV-negative patients. UpToDate (last access May 18, 2020) Available from: https://www.uptodate.com/contents/over view-of-nontuberculous-mycobacterialinfections? search $=$ overview $\% 20 \mathrm{of} \% 20 \mathrm{n}$ ontuberculous $\% 20$ mycobacterial $\% 20 \mathrm{inf}$ ections $\% 20 \mathrm{in} \% 20$ Immunocompromised $\% 20$ host $\% 20$ negative $\% 20$ patients\&sour ce $=$ search_result\&selectedTitle $=1 \sim 150$ \&usage_type $=$ default\&display_rank $=1$

2. Daley $\overline{C L}$, Kasperbauer S. Treatment of lung infection with Mycobacterium kansasii and other less common nontuberculous mycobacteria in adults. UpToDate (last access May 18, 2020). Available from: https://www.uptodate. com/contents/treatment-of-lunginfection-with-mycobacterium-kansasiiand-other-less-common-nontuberculousmycobacteria-in-adults?search=.\%20Tre atment $\% 20$ of $\% 20$ lung $\% 20$ infection $\% 20$ with $\% 20$ Mycobacterium $\% 20$ kansasii\% 20and\%20other\%20less $\% 20$ common $\%$ 20nontuberculous $\% 20$ mycobacteria $\% 20$ in $\% 20$ adults\&source=search_result\&sel ectedTitle $=1 \sim 150 \&$ usage_type $=$ default \&display_rank $=1$

3. Bae JY, Lee JI, Kim HL, et al. Disseminated tuberculosis following adalimumab treatment in psoriasis despite negative screening. Int $\mathrm{J}$ Dermatol 2019; 58:98-100.

4. Bekou V, Büchau A, Flaig MJ, et al. Cutaneous infection by Mycobacterium haemophilum and kansasii in an IgAdeficient man. BMC Dermatol 2011; 11:3.

5. Blue ML, Payne WG, Mannari RI, et al. Mycobacterium kansasii causing carpal tunnel syndrome with concomitant pulmonary Mycobacterium tuberculosis infection. South Med J 2002; 95:1095-8.

6. Min Z, Amlani M. Pulmonary Mycobacterium kansasii Infection Mimicking Malignancy on the (18) FFDG PET Scan in a Patient Receiving Etanercept: A Case Report and Literature Review. Case Rep Pulmonol 2014;2014:973573.

7. Harris J, Keane J. How tumor necrosis factor blockers interfere with tuberculosis immunity. Clin Exp Immunol 2010;161:1-9.

8. Winthrop KL, Yamashita S, Beekmann SE, Polgreen PM. Infectious Diseases Society of America Emerging Infections Network. Mycobacterial and other serious infections in patients receiving anti-tumor necrosis factor and other newly approved biologic therapies: case finding through the Emerging Infections Network. Clin Infect Dis 2008;46:173840.

9. Griffith DE, Aksamit T, Brown-Elliott BA, et al. An official ATS/IDSA statement: diagnosis, treatment, and prevention of nontuberculous mycobacterial diseases. Am J Respir Crit Care Med 2007;175:367.

10. Kobashi Y, Mouri K, Yagi S, et al. Clinical evaluation of the QuantiFERON-TB Gold test in patients with non-tuberculous mycobacterial disease. Int $\mathrm{J}$ Tuberc Lung Dis 2009;13:1422-6.

11. Panés-Rodríguez A, Vildósola-Esturo S, Rodríguez-Pérez I, et al. Reddish nodule on the hand. A Quiz. Acta Derm Venereol 2016; 96:1004-8.

12. Simon A, Onya O, Mazza-Stalder J, et al. Added diagnostic value of $16 \mathrm{~S}$ rRNA gene pan-mycobacterial PCR for nontuberculous mycobacterial infections: a 10-year retrospective study. Eur J Clin Microbiol Infect Dis 2019;38:1873-81.

13. Bartralot R, García-Patos V, Sitjas D, et al. Clinical patterns of cutaneous nontuberculous mycobacterial infections. Br J Dermatol 2005; 152:72734.

14. Fernández-Guerrero ML, Esteban J, Acebes C, Górgolas M. Failure of Isoniazid Chemoprophylaxis During Infliximab Therapy. Emerg Infect Dis 2007;13:1428-9.

15. Spiliopoulou I, Foka A, Bounas A, Marangos MN. Mycobacterium kansasii cutaneous infection in a patient with sarcoidosis treated with anti-TNF agents. Acta Clin Belg 2014;69:229-31.

16. Malkin J, Shrimpton A, Wiselka M, et al. Olecranon bursitis secondary to Mycobacterium kansasii infection in a patient receiving infliximab for Behcet's disease. J Med Microbiol 2009;58:3713.

17. Hsu PY, Yang YH, Hsiao $\mathrm{CH}$, et al. Mycobacterium kansasii infection presenting as cellulitis in a patient with systemic lupus erythematosus. J Formos Med Assoc 2002; 101:581-4.

18. Crowley J, Thaçi D, Joly P, et al. Longterm safety and tolerability of apremilast in patients with psoriasis: Pooled safety analysis for $\geq 156$ weeks from 2 phase 3 , randomized, controlled trials (ESTEEM 1 and 2). J Am Acad Dermatol 2017;77:310-317. 